\title{
Avaliação laboratorial da classificação de Kennedy e dos tipos de retentores protéticos utilizados em uma cidade do sertão paraibano
}

\author{
Laboratory evaluation of Kennedy classification and types of protetical retinators used a city of sertão paraibano \\ (northeastern of Brazil)
}

Evaluación laboratorial de la clasificación de Kennedy y de los tipos de retentores proteticos utilizados en una ciudad del sertón paraibano

\author{
Ana Karina Almeida ROLIM ${ }^{\mathbf{1}}$ \\ Maria Luiza Leite dos SANTOS ${ }^{2}$ \\ Thássio Sousa de OLIVEIRA2 \\ Carlus Alberto Oliveira dos SANTOS ${ }^{1}$ \\ Rachel de Queiroz Ferreira RODRIGUES ${ }^{2}$ \\ Rodrigo Araújo RODRIGUES ${ }^{2}$
}

${ }^{I}$ Programa de pós-graduação em Odontologia, Universidade Estadual da Paraíba - UEPB, 58431-000, Campina Grande, Paraíba, Brasil

${ }^{2}$ Departamento de Odontologia da Universidade Federal de Campina Grande, UFCG, 58700-000, Patos, Paraíba, Brasil

\section{Resumo}

Introdução: A reabilitação oral dos pacientes parcialmente dentados pode ser realizada através de próteses parciais removíveis (PPRs) e constitui fator essencial para a manutenção da qualidade de vida. Objetivo: Identificar quais retentores são mais utilizados nas próteses parciais removíveis da cidade de Patos-PB. Metodologia: Foram selecionados 5 laboratórios de prótese dentária e nestes avaliados 10 modelos em gesso da arcada superior e 10 da arcada inferior, bem como as próteses parciais removíveis confeccionadas para cada modelo. As PPRs foram examinadas uma por uma quanto a sua Classificação seguindo Kennedy e quais os retentores encontrados. Resultados: A Classe III, Mod 1 de Kennedy foi a classe encontrada com maior prevalência na maxila (24\%), seguida da Classe III, Mod 2 (22\%). Enquanto que na mandíbula, a Classe mais prevalente foi a Classe I (36\%). Em relação aos retentores, foram analisados 201 grampos na maxila sendo o mais prevalente o grampo de abraçamento Circunferencial Simples (32\%). Na mandíbula foram encontrados 191 retentores e o grampo de ação de ponta tipo "T" (40\%) foi o mais prevalente. Conclusão: A Classificação de Kennedy mais encontrada em PPRs, da cidade de Patos, foram a Classe III, Mod 1 na maxila e a Classe I, na mandíbula e os retentores mais utilizados, foram os circunferenciais simples na maxila e o grampo de ação de ponta do tipo "T" na mandíbula.

Descritores: Odontologia; Prótese Parcial Removível; Grampos Dentários.

\section{Abstract}

The objective of the present study is to identify which retainers are most used in removable partial dentures at the city of Patos, PB, Brazil. Five dental laboratories were selected at the city of Patos, Paraíba. Data collection was held at unannounced visits. 10 models of the upper arch and 10 of the lower arch submitted by dentists were evaluated in each laboratory. The RPDs were cataloged, examined one by one and the reference data. Their classification following Kennedy and the retainers used were added to Microsoft Excel® 2007 and a descriptive statistics were realized. In this study 100 metal frames of removable partial dentures and their models and models were submitted, being 50 maxillaries and 50 mandibular. A Class III, Mod 1 of Kennedy for a class found with higher prevalence in the maxilla (24\%), Class III, Mod $2(22 \%)$. While in the mandible, a Class more prevalent for a Class I (36\%). In relation to the retainers, 201 clamps were analyzed in the maxilla being the most prevalent or Simple Circunferencial clamping clamp (32\%). In the mandible, 191 retainers were found and the "T" tipped action clamp (40\%) was the most prevalent. Was concluded that the Kennedy classification most found in RPDs, at the city of Patos, were Class III, Mod 1 in the maxilla and Class I, in the mandible and the most used retainers, in the maxilla were the simple circumferential and in the jaw the "T" tipped action clamp.

Descriptors: Dentistry; Denture, Partial, Removable; Dental Clasps.

\section{Resumen}

Introducción: la rehabilitación oral de pacientes parcialmente dentados se puede realizar con prótesis parciales removibles (PPR) y es un factor esencial para mantener la calidad de vida. Objetivo: identificar qué retenedores son los más utilizados en prótesis parciales removibles en la ciudad de Patos-PB. Metodología: se seleccionaron cinco laboratorios de prótesis dentales y en estos se evaluaron 10 modelos de yeso del arco superior y 10 del arco inferior, así como las prótesis parciales removibles hechas para cada modelo. Los PPR se examinaron uno por uno con respecto a su clasificación según Kennedy y qué retenes se encontraron. Resultados: Kennedy Clase III, Mod 1 fue la clase con mayor prevalencia en el maxilar (24\%), seguido de Clase III, Mod 2 (22\%). Mientras que en la mandíbula, la clase más frecuente fue la clase I (36\%). Con respecto a los retenedores, se analizaron 201 pinzas en el maxilar, siendo la más frecuente la pinza circunferencial simple (32\%). Se encontraron 191 retenedores en la mandíbula y el clip de acción tipo "T" (40\%) fue el más frecuente. Conclusión: La Clasificación Kennedy más encontrada en PPR, en la ciudad de Patos, fue Clase III, Mod 1 en el maxilar y Clase I, en la mandíbula y los retenedores más utilizados, fueron la circunferencial simple en el maxilar y la pinza de acción de Punta tipo "T" en la mandíbula.

Descriptores: Odontología; Dentadura Parcial Removible; Abrazadera Dental.

\section{INTRODUÇÃO}

A reabilitação oral dos pacientes parcialmente dentados pode ser realizada através de próteses parciais removíveis (PPRs) e constitui fator essencial para a manutenção da qualidade de vida ${ }^{1}$. As PPRs são bastante requisitadas no intuito de repor os dentes naturais perdidos e estruturas associadas. Uma correta avaliação, diagnóstico diferencial e planejamento detalhado devem ser realizados antes da confecção desse aparelho para que haja um maior sucesso no trabalho realizado ${ }^{2}$.

A execução de uma PPR é relativamente simples, efetiva e de baixo custo ${ }^{3}$. Entretanto, o aparelho é de difícil aceitação pelos pacientes, visto que são volumosas, desconfortáveis e instáveis, principalmente se tiverem extremidade livre ${ }^{4}$. Entre as desvantagens no uso da PPR tem-se o maior acúmulo de biofilme nos braços dos retentores, dificuldade de higienização pelo usuário, inflamação gengival e até mesmo ocorrência de perda de inserção óssea, recessão gengival e cárie nos dentes pilares.

Para obtenção de uma maior retenção da prótese deve-se tomar mão de estruturas retentoras, como por exemplo, os grampos utilizados nessas 
PPRs. Estes, por sua vez, são estruturas confeccionas a partir de ligas metálicas e que na maioria das vezes são feitas por cobalto e cromo. A partir de sua introdução na odontologia as ligas de cobalto e cromo (Co-Cr) passaram a substituir as ligas nobres na confecção das PPRs, até tornarem-se as mais comumente empregadas para a fundição deste tipo de prótese $^{6}$.

A cidade de Patos, no estado da Paraíba, Brasil, por ser destaque com o seu desenvolvimento econômico comparado às demais cidades do sertão paraibano e, por ter sua localização geográfica no centro da região, torna-se a cidade mais requisitada pelos habitantes das cidades circunvizinhas, favorecendo o seu desenvolvimento comercial. Consequentemente, um maior número de laboratórios odontológicos é instalado para atender as necessidades do município de Patos e região. Logo, aproveitando o ambiente propenso para a pesquisa elaborada, este trabalho objetivou avaliar quais os retentores mais utilizados em PPRs pelos laboratórios protéticos da cidade e qual classificação de Kennedy mais frequentemente encontrada.

\section{MATERIAL E MÉTODO}

Esta é uma pesquisa do tipo observacional, de abordagem quantitativa, analítica e documental. Foram selecionados 5 laboratórios de prótese dentária da cidade de Patos, Paraíba, seguindo metodologia utilizada por Pun et al. ${ }^{7}$. Para inclusão, foram seguidos os seguintes critérios: o laboratório deveria realizar fundição de armações metálicas em Co-Cr e o Responsável Técnico deveria possuir registro no Conselho Regional de Odontologia. Além disso, por meio de Termo de Consentimento Livre e Esclarecido, deveria permitir a realização de fotografias dos modelos e armações metálicas confeccionadas.

A coleta dos dados foi realizada em visitas sem aviso prévio aos laboratórios selecionados e foram avaliados em cada laboratório, 10 modelos em gesso da arcada superior e 10 da arcada inferior enviados pelos dentistas de consultórios odontológicos da cidade de Patos. Não poderia haver qualquer indício de identificação tanto para o profissional quanto para o paciente.

As PPRs foram catalogadas, examinadas uma por uma e os dados referentes à sua Classificação seguindo Kennedy e quais os retentores encontrados foram adicionados ao Microsoft Excel® 2007 e realizado a estatística descritiva.

Os modelos em gesso foram fotografados a fim de obter a visualização imediata das arcadas parcialmente desdentadas, registrando todas as superfícies oclusais e incisais, para posterior classificação de todos os modelos de acordo com a classificação topográfica universal de Kennedy, com as regras de Applegate aplicadas. Além disso, foram fotografadas as respectivas armações metálicas em ligas de $\mathrm{Co}-\mathrm{Cr}$, para análise dos retentores mais prevalentes.

A Classificação de Kennedy, com regras de Applegate, possui 4 divisões, algumas com subclassificações. A classe I apresenta pacientes sem dentes posteriores bilaterais; Classe II são os casos unilaterais; Classe III são os pacientes edêntulos unilaterais posteriores que possuem dentes pilares nesta região e por último a classe IV que são os pacientes edêntulos na região anterior, esta é a única classe que não apresenta modificações.

A avaliação específica dos retentores foi realizada seguindo a classificação quanto à forma e origem do braço de retenção em grampos circunferenciais simples, circunferenciais em anel, ação de ponta tipo "T", T modificado, ação de ponta tipo "I", Mesio-disto-lingual (MDL) e Continuo de Kennedy.

\section{RESULTADOS}

Neste estudo foram avaliados 100 armações metálicas de próteses parciais removíveis e seus respectivos modelos, sendo 50 maxilares e 50 mandibulares.

A Classe III, Mod 1 de Kennedy foi a classe encontrada com maior prevalência na maxila (24\%), seguida da Classe III, Mod 2 (22\%). A classe IV foi a de menor prevalência neste estudo, representando apenas $6 \%$ do total. Na mandíbula, a Classe mais prevalente foi a Classe I (36\%), seguida da Classe I, Mod 1 (20\%) e Classe III, Mod 1 (18\%), respectivamente (Tabela 1).

Tabela 1. Classificação de Kennedy e suas modificações na arcada superior e inferior

\begin{tabular}{l|c|c|c|c|}
\hline \multirow{2}{*}{ CLASSIFICAÇÃO } & \multicolumn{2}{|c}{ SUPERIOR } & \multicolumn{2}{c}{ INFERIOR } \\
\hline I & $\mathrm{n}$ & $\%$ & $\mathrm{n}$ & $\%$ \\
\hline I, MOD I & 1 & 2 & 18 & 36 \\
I, MOD II & 5 & 10 & 10 & 20 \\
I, MOD III & 0 & 0 & 0 & 0 \\
II & 0 & 0 & 0 & 0 \\
II, MOD I & 0 & 0 & 0 & 0 \\
II, MOD II & 2 & 4 & 2 & 4 \\
II, MOD III & 6 & 12 & 5 & 10 \\
III & 6 & 12 & 0 & 0 \\
III, MOD I & 0 & 0 & 0 & 0 \\
III, MOD 2 & 12 & 24 & 9 & 18 \\
III, MOD 3 & 11 & 22 & 5 & 10 \\
IV & 4 & 8 & 0 & 0 \\
\hline TOTAL & 3 & 6 & 1 & 2 \\
\hline
\end{tabular}

Quando relacionamos as arcadas dentárias com a classificação de Kennedy, em ambos os maxilares encontramos uma predominância de desdentados Classe III e suas modificações (41\%), seguidos de Classe I e suas modificações (34\%) e Classe II e suas modificações (21\%). A Classe IV (4\%) é a menos encontrada em ambas as arcadas.

Foram analisados 200 grampos na maxila. Dentre estes, foi encontrado de forma mais prevalente o grampo de abraçamento Circunferencial 
Simples (32\%). O grampo de ação de ponta tipo "I" não foi encontrado e os retentores T-modificado (3\%), Contínuo de Kennedy (1\%) e Circunferencial em Anel (1\%) apresentaram baixa prevalência. Na mandíbula, foram encontrados 189 retentores e o grampo de ação de ponta tipo "T" $(40 \%)$ foi o mais prevalente, seguido pelos Circunferenciais Simples (25\%) (Tabela 2).

Tabela 2. Retentores utilizados em próteses parciais removíveis superiores e inferiores

\begin{tabular}{|l|c|c|c|c|}
\hline RETENTORES & $\mathbf{N}$ & $\mathbf{\%}$ & $\mathbf{N}$ & $\mathbf{\%}$ \\
\hline AÇÃO DE PONTA “T” & 58 & 29 & 76 & 40 \\
AÇÃO DE PONTA “T” MOD & 6 & 3 & 11 & 6 \\
AÇÃO DE PONTA “I” & 0 & 0 & 0 & 0 \\
CIRCUNFERENCIAL SIMPLES & 64 & 32 & 48 & 25 \\
CIRCUNFERENCIAL GEMINADO & 18 & 9 & 3 & 2 \\
CIRCUNFERENCIAL ANÉL & 2 & 1 & 0 & 0 \\
MDL & 50 & 25 & 38 & 20 \\
CONTÍNUO DE KENNEDY & 2 & 1 & 13 & 7 \\
\hline TOTAL & 200 & 100 & 189 & 100 \\
\hline
\end{tabular}

Ao analisar, quais os retentores mais utilizados, no total, ou seja, em ambas as arcadas, constatou-se que o grampo de ação de ponta do tipo "T" (34\%) era o mais frequente, seguido do Circunferencial Simples (29\%).

\section{DISCUSSÃO}

A Odontologia tem assumido, com o passar dos anos, um caráter de promoção e prevenção de saúde bucal. Entretanto, ainda pode-se verificar uma alta prevalência de perdas dentárias. Isto se deve em grande parte as características culturais da população brasileira, que acredita que as perdas dentárias são inevitáveis e que ocorrem com o passar da idade. Em decorrência disto, muitos dentes são precoce e desnecessariamente extraídos sendo substituídos por próteses dentárias ${ }^{8}$.

As PPRs têm como objetivo principal restabelecer o sistema estomatognático e devolver ao paciente características físicas e fisiológicas. Para uma reabilitação eficaz, a PPR precisa ser bem planejada pelo cirurgião-dentista, levando em consideração todas as características oclusais, funcionais e estéticas bem como, todas as limitações do paciente em questão ${ }^{3}$.

É de fundamental importância pesquisas que identifiquem quais as classificações prevalentes de arcadas parcialmente desdentadas, pois com base nos dados é possível elaborar programas preventivos de ações locais e minimizar as situações decorrentes das ausências dentárias e até mesmo estudar biomecanicamente as alternativas mais viáveis para elaborar planejamentos dessa modalidade de tratamento reabilitador ${ }^{9}$.

Souza et al. $^{10}$, ao avaliarem o número e a classificação de Kennedy das arcadas parcialmente desdentadas em pacientes tratados com PPRs, no Rio de Janeiro, entre 2005 e 2010, observou que a Classe I de Kennedy foi a mais frequente na arcada mandibular enquanto a Classe III foi a que mais predominou na maxila. Pun et al. $^{7}$, ao realizarem estudo em 5 laboratórios dentários de Milwaukee, constataram que entre os 1502 modelos fotografados, a Classe I mantêm-se mais frequente na mandíbula, enquanto que na maxila a maior frequência é de Classes III. Além disso, ao avaliar o total, ou seja, ambas as arcadas, observou-se que há uma maior frequência de Classe III, o que se pode explicar pelo fato de que os cuidados em saúde bucal estão melhorando e consequentemente uma redução da perda dos dentes também. Entretanto, deve-se sempre há o alerta para o desenvolvimento de estratégias de promoção e prevenção da saúde bucal e da necessidade da reabilitação oral.

Outro estudo realizado por Duarte e Paiva ${ }^{11}$, nas cidades de Recife, Natal e João Pessoa também revelou maior frequência para a classe III $(44,17 \%)$, seguidas das classes I $(26,04 \%)$, II (25\%) e IV $(4,79 \%)$ respectivamente. Alencar et al. $(2016)^{12}$, avaliando o planejamento de prótese parciais removíveis em modelos recebidos pelos laboratórios de Teresina, Piauí, constataram que há naquele local uma maior prevalência de arcos parcialmente edentados de Classe III com alguma modificação não especificada (35\%), seguido da Classe I sem modificação (20\%).

Já em uma pesquisa realizada na Paraíba envolvendo as cidades consideradas polos econômicos regionais, que avaliou a classificação de Kennedy mais prevalente, obteve como resultados que em João Pessoa foi encontrado maior percentual de desdentados para classe II $(34,7 \%)$. Enquanto que em Campina Grande, Patos e Sousa houve predominância de classe III. Para a classe IV, percentual abaixo de $8 \%$ ocorreram para as 4 cidades. Apenas em Cajazeiras ocorreu resultado superior $(14,4 \%)^{13}$. Tais resultados concordam com este estudo, onde há em maior ocorrência próteses classificadas em Classe III, em detrimento da Classe IV, que apresenta baixa prevalência.

Os grampos são componentes das PPR que tem a função de resistir às forças de deslocamentos a elas aplicadas, oferecendo suporte, retenção e estabilidade, além de preservar as estruturas de suporte relacionadas a estas, direta ou indiretamente ${ }^{14}$.

No presente estudo, ao analisar ambas as arcadas, observou-se que os grampos por ação de ponta " $T$ " foram os mais usados como retentores. Estes grampos, em virtude de suas características físicas e sua constituição (são mais longos, flexíveis, retentivos e aproximam-se dos dentes pela cervical), permitem um grau aceitável de movimentação da prótese diminuindo o torque sobre os dentes suportes ${ }^{15}$.

Os que apresentaram o segundo maior percentual de ocorrência, em ambas as arcadas, foram os Circunferenciais Simples. Conforme Pezzoli et 
al. ${ }^{16}$, estes grampos são frequentemente utilizados em próteses dento-suportadas devido a sua capacidade de conferir retenção e abraçamento adequado. Já em PPRs de extremidade livre, os grampos circunferências simples, apresentam comportamento biomecânico desfavorável em comparação aos grampos por ação de ponta.

\section{CONCLUSÃO}

A Classificação de Kennedy mais encontrada em PPRs, na cidade de Patos, na Paraíba, foi a Classe III, Mod 1 na maxila e a Classe I, na mandíbula. Os retentores mais utilizados, na maxila foram os Circunferenciais Simples e na mandíbula o grampo de ação de ponta do tipo "T".

\section{REFERÊNCIAS}

1. Carreiro AFP, Bezerra CFR, Amaral BA, Piuvezam G, Seabra EG. Aspectos biomecânicos das próteses parciais removíveis e o periodonto de dentes suporte. R Periodontia. 2008;18(1):105-13.

2. Meyer GA, Schindler JM, Urbanetto CR, Leon BLT. Avaliação dos planejamentos realizados por técnicos em prótese dentária em modelos Classe I de Kennedy. Rev Bahiana Odonto. 2012;3(1):26-36.

3. Ribeiro SDO, Albuquerque ACLD, Rodrigues RA, Santos PPDA. Relação entre desordens temporomandibulares (dtm) e pacientes portadores de próteses parciais removíveis. Odontol Clín Cient. 2015;14(1):565-70.

4. Carvalho W, Silva SRB, Barboza ESP, Gouveia CVD. Prótese parcial removível retida por implantes e dente em maxila parcialmente edêntula. RGO. 2006;54(3):244-48.

5. Flora CD, Sensever FA, Skupien JA, Zanatta FB, Antoniazzi RP. Condições periodontais de usuários de prótese parcial removível. Disciplinarum Scientia. 2017;18(3):489-500.

6. Kliemann C, Oliveira W. Manual de Prótese Dentária. São Paulo: Santos; 1996.

7. Pun DK, Waliszewski MP, Waliszewski KJ, Berzins D. Survey of partial removable dental prosthesis (partial RDP) types in a distinct patient population. J Prosth Dent. 2011;106(1):48-56.

8. Azevedo JS. SB BRASIL 2010: Uso e necessidade de prótese dentária em idosos. [dissertação]. Pelotas (RS): Universidade Federal de Pelotas; 2014.

9. Zavanelli RA, Guilherme AS, Tavares LR. Prevalência de arcadas parcialmente desdentadas de pacientes atendidos na Faculdade de Odontologia-UFG de 1994 a 2004. Robrac. 2007;16(42):23-27.

10. Souza FN, Gomes CS, Rodrigues ARC, Tiossi R, Gouvêa CVD, Almeida CC. Partially edentulous arches: a 5-year survey of patients treated at the Fluminense Federal University Removable Prosthodontics Clinics in Brazil. J Prosthod. 2015;24(6):447-51.
11.Duarte ARC, Paiva HJ. Avaliação do nível de conhecimento e conscientização do cirurgiãodentista e do técnico de prótese dental, em relação ao planejamento e execução de próteses parciais removíveis. Rev ABO Nac. 2000;8(4):232-37.

12. Alencar GXD, Pedrosa MDS, Lopes LDS. Avaliação do planejamento em modelos para próteses parciais removíveis recebidos por laboratórios de Teresina, Piauí. Salusvita. 2016;35(3):423-35.

13.Araujo TP, Gonçalves CJS, Bezerra ALT, Cruz DF, Fernandez ML, Mukai MK et al. Prevalência dos tipos de arcos desdentados, preparo de boca e qualidade dos modelos para próteses removíveis na Paraíba. Rev bras ciênc saúde. 2012; 16(2):213-18.

14.Zlataric DK, Celebric A, Valentic-Peruzovic M. The effect of removable partial dentures on periodontal health of abutment teeth. J Periodontol. 2002;73(2):137-44.

15. Todescan R, Silva EEB, Silva OJ. Atlas de prótese parcial removível. São Paulo: Santos; 1996.

16.Pezzoli M, Appendino P, Calcagno L, Celasco M, Modica R. Load transmission evaluation by removable distal-extension partial dentures using holographic interferometry. J Dent. 1993; 21(5):312-16.

\section{CONFLITO DE INTERESSES}

Os autores declaram não haver conflitos de interesse.

AUTOR PARA CORRESPONDENCIA

\section{Ana Karina Almeida Rolim}

k_rolim19@hotmail.com

Submetido em 06/02/2019

Aceito em 24/05/2019 